\begin{tabular}{|c|c|}
\hline Title & Semi-Three-Dimensional V isualization of Electromagnetic Field A nalysis Result With V olumetric Display \\
\hline Author(s) & Noguchi, So; Inaba, Tomoaki; Igarashi, Hajime \\
\hline Citation & $\begin{array}{l}\text { IEEE Transactions on Magnetics, } 47(5), 1330-1333 \\
\text { https://doi.org/10.1109/ MA G.2010.2089437 }\end{array}$ \\
\hline Issue Date & 2011-05 \\
\hline Doc URL & http://hdl.handle.net/2115/45426 \\
\hline Rights & $\begin{array}{l}\text { (c) } 2011 \text { IEEE. Personal use of this material is permitted. Permission from IEEE must be obtained for all other uses, in } \\
\text { any current or future media, including reprinting/republishing this material for advertising or promotional purposes, } \\
\text { creating new collective works, for resale or redistribution to servers or lists, or reuse of any copyrighted component of } \\
\text { this work in other works. }\end{array}$ \\
\hline Tyре & article (author version) \\
\hline File Information & ToM 47-5_1330-1333.pdf \\
\hline
\end{tabular}

Instructions for use 


\title{
Semi-three-dimensional Visualization of Electromagnetic Field Analysis Result with Volumetric Display
}

\author{
So Noguchi, Tomoaki Inaba, and Hajime Igarashi \\ Graduate School of Information Science and Technology, Hokkaido University \\ Kita 14 Nishi 9, Kita-ku, Sapporo 060-0814, Japan
}

\begin{abstract}
When visualizing a 3-D electromagnetic field analysis result, physical quantity on a surface or a cross-section of objects is visualized mostly. It is, however, desired to observe the physical quantity and the phenomenon inside the objects in 3-D space. In this paper, the method to visualize the physical quantity inside the objects with a volumetric display is proposed. The volumetric display is based on a stack of LCDs, and developed to separately display the images on the LCDs. With the volumetric display, the physical quantity, which changes on different cross-sections of the objects in 3-D, can be visualized simultaneously.

The volumetric display is also used for image comparison. In order to compare the results of magnetic field analysis, usually each image is compared by overlappingly visualizing these. However, it is easy to visualize the images and compare these, using the volumetric display.
\end{abstract}

The volumetric display is a useful tool for post-processing of the electromagnetic field analysis.

Index Terms - Electromagnetic field analysis, scientific visualization, volumetric display

\section{INTRODUCTION}

W ITH IMPROVEMENT of computer performances, a large scale analysis on electromagnetic field is often performed. The magnetic devices, such as massive conductors in series connection, a permanent magnet motor, a transformer, an induction motor and so on, are commonly analyzed by 3-D advanced simulation techniques [1], [2]. After the analysis, it is difficult to three-dimensionally observe the phenomenon since the huge amount of data is generated from the analysis. Hence, various techniques for visualizing the magnetic field in 3-D have been proposed [3]-[5]. The visualization of magnetic flux line in 3-D space is the most promising method, but a good method depicting the magnetic flux lines hasn't been established yet. Recently, the stereoscopic 3-D monitor without the use of any special glasses [6] is used for visualizing the physical quantity of the 3-D finite element analysis result. The stereoscopic 3-D monitor, however, visualizes the surface of the objects in 3-D. Therefore, it is difficult to observe the detail of the phenomenon inside the objects.

After the 3-D finite element analysis, e.g. a skewed motor and a reluctance motor [1]-[3], is performed, it is desired to observe the phenomenon inside the objects in the longitudinal direction. That is, the magnetic field inside the rotor and the stator is visualized to check the skew effect. In common, we have to depict the vector field maps on many cross-sections in order to observe and compare these maps. Therefore, in this study, the vector field maps on a few cross-sections are simultaneously visualized on the volumetric display [7]. As the results, it is easy to observe the detail of the magnetic field inside the object and to compare a few analysis results. The purpose of this study is to develop the volumetric display and to apply it to visualizing the physical quantities changing in the longitudinal direction, such as a skew effect.

Manuscript received May 31, 2010. Corresponding author: S. Noguchi (e-mail: noguchi@ssi.ist.hokudai.ac.jp).

Digital Object Identifier inserted by IEEE

\section{VOLUMETRIC DISPLAY}

The volumetric display is one of three-dimensional display technologies that produce volume-filling imagery such as DepthCube [8], Perspecta [9]. The images on the volumetric display, unlike on the stereoscopic 3-D monitor, are displayed in a real 3-D space. Therefore, the volumetric display can show the 3-D image to an observer without visually induced motion sickness. The cause of the motion sickness is that the distance perceived in his/her brain is different from the real distance. As a result, using the volumetric display, it is possible to observe the images in 3-D without an illusion.

Fig. 1 shows the conceptual illustration of the volumetric display. The volumetric display consists of a rear LCD with a light source and some front LCDs with high transmissivity. An image on the LCD with high transmissivity is clear with less lightness. Images are displayed on every LCD, an observer overlappingly watch all the images, even the image on the rear LCD.

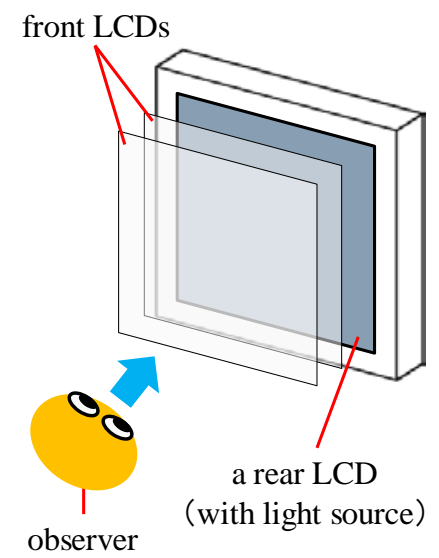

Fig. 1. The concept of volumetric display. Front LCDs are highly transmissive, and a rear LCD is a normal LCD with a light source. 
The developed volumetric display consists of a rear LCD with a light source (I-O DATA product LCD-AD19H) and a front LCD with high transmissivity (DISIGN product R120F00-M1TR), as shown in Fig. 2. It is necessary that the front LCD is selected under considering the direction of polarization filter. The images on the rear and the front LCDs are simultaneously and overlappingly displayed. The volumetric display is effective in the following use. First one is a semi-three-dimensional visualization displaying the multiple cross-sections inside the object on each LCD, described in section III. Another one is an image comparison to observe the difference between the images on each LCD, described in section IV.

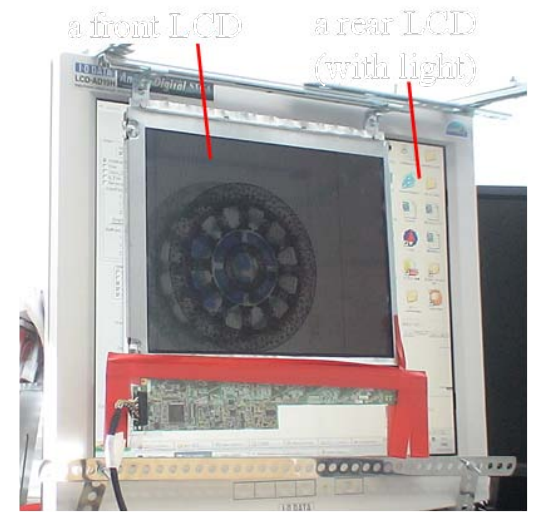

Fig. 2. The developed volumetric display. The rear LCD is an I-O DATA product LCD-AD19H. The front LCD is a DISIGN product R120F00M1TR, that is sold by the usage in outdoor.

\section{SEMI-3-D VisUALIZATION}

In visualization of 3-D analysis results, physical quantity distribution on a surface or a cross-section is visualized commonly. It is, however, desirable that the distributions on multiple cross-sections inside the object are simultaneously visualized to clearly observe a skew effect or a coil end effect. With the volumetric display, it is possible to visualize the different cross-sections on every LCD. Since the distance from eyes' point to each LCD is different, the user can perceive the distance to the focused LCD. He/she can clearly observe the image on the focused LCD, on the other hand, the images on the not-focused LCDs are dim and unclear, as shown in Fig. 3. So the user can perceive the distance to the image on the focused LCD. This distance in vision gives a semi-threedimensional view to the user. In addition, by adjusting the distance between the LCDs, various semi-three-dimensional views are effectively given to the user.

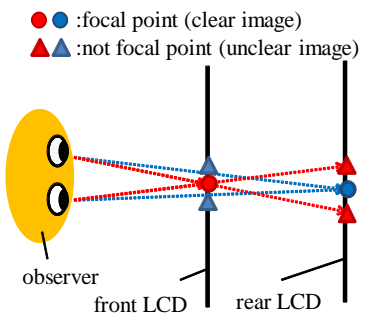

Fig. 3. The observer can clearly perceive the image on the focused LCD, but he/she dimly and unclearly see the image on the not-focused LCD. He/she can perceive the distance to the image on the focused LCD.
Fig. 4 shows a surface permanent magnet motor with skew, which is used to observe the magnetic field changing in the longitudinal direction. The magnetic field analysis was done by the edge finite element method. The magnetic field vector maps on two arbitrary cross-sections, that are in the partial area enclosed with the circle in Fig. 4, are shown in Fig. 5. The cross-sections are near the front surface and the back surface of the surface permanent magnet motor, respectively. The two magnetic field vector maps are different due to the skewing permanent magnet. By watching the two images of Fig. 5, we can't perceive the three-dimensional view, even if the two images are overlapped.

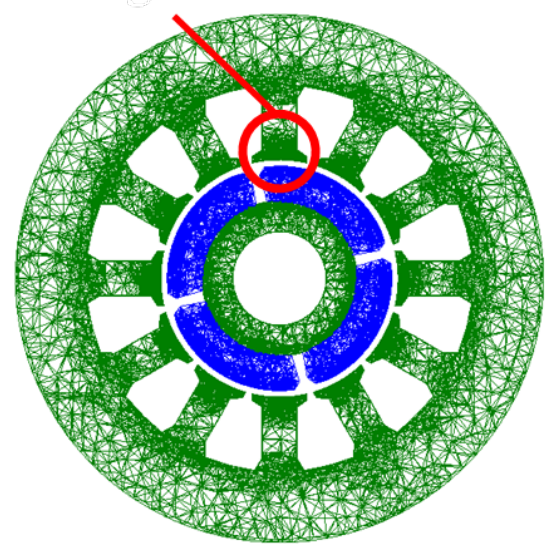

Fig. 4. The surface permanent magnet motor model with skew, which is used to observe the magnetic field changing in the longitudinal direction.

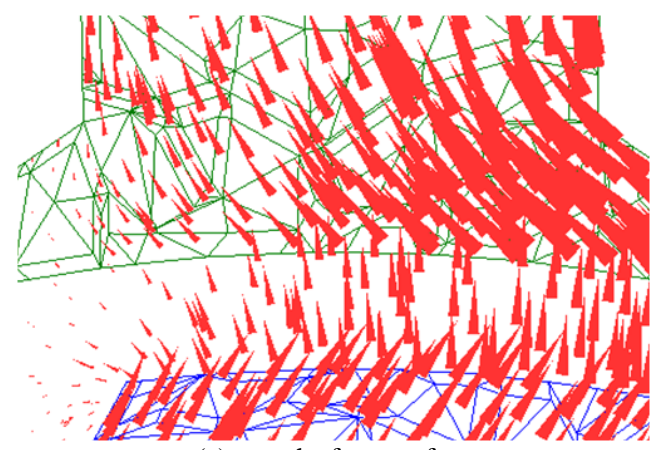

(a) near the front surface

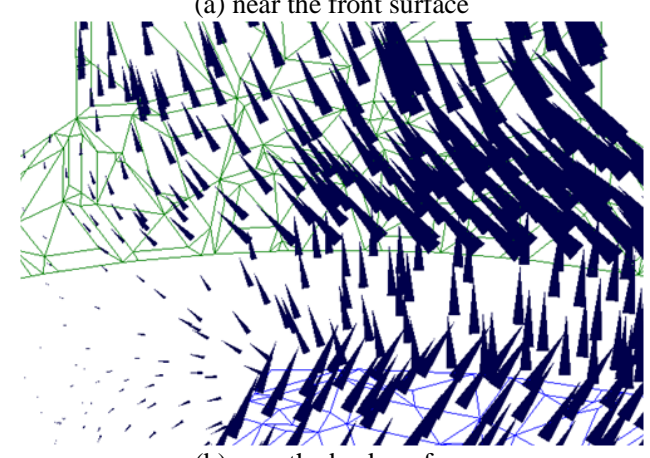

(b) near the back surface

Fig. 5. The magnetic field vector maps on the cross-sections, which are near the front surface and the back surface of the permanent magnet motor. The magnetic field vector maps are different due to the skew. 
To give the three-dimensional view, the images on Figs. 5(a) and (b) are visualized on the front and the rear LCDs of the developed volumetric display, respectively. Fig. 6(a) shows the picture when focusing the front LCD, so the red cones are clear but the blue ones are dim and unclear. On the other hand, Fig. 6(b) shows the picture when focusing on the rear LCD, so the blue cones are clear but the red ones are dim and unclear. Consequently, the observer can perceive the distance between the front and the rear LCDs. In addition, the magnetic field changing in the longitudinal direction inside the motor can be observed well. It is possible to recognize that the direction of some red cones in the air gap is obviously different from that of the blue ones.

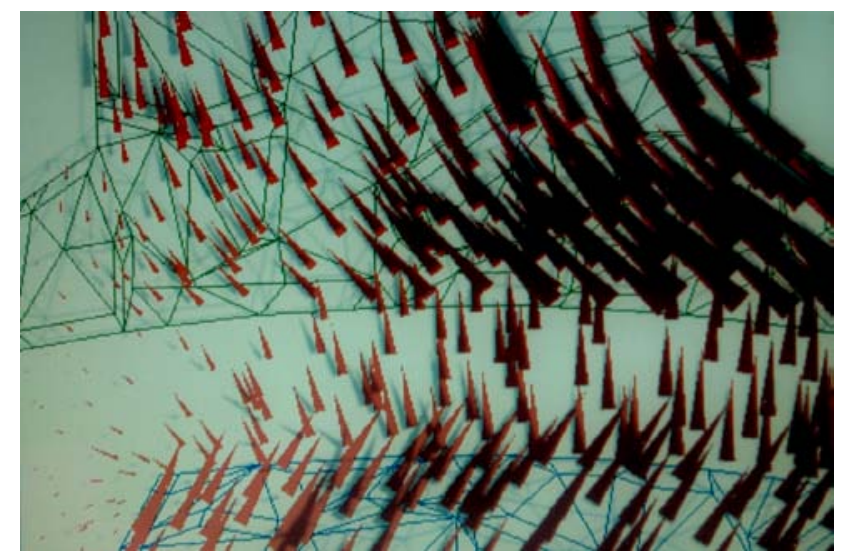

(a)

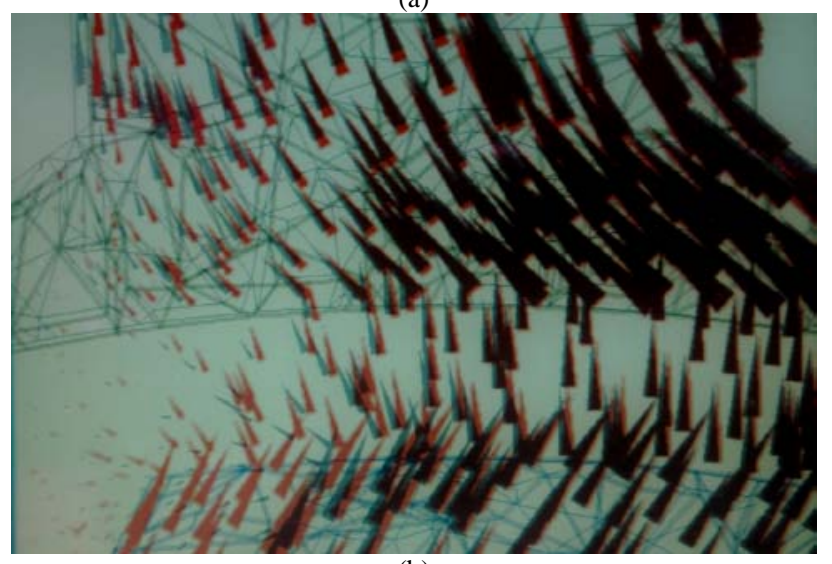

(b)

Fig. 6. The magnetic field vector maps with the volumetric display. (a) Focusing on the front LCD, the red cones are clear but the blue ones are dim and unclear. On the other hand, focusing on the rear LCD, the blue cones are clear but the red ones are dim and unclear. Consequently, the observer can perceive the distance with the font and the rear LCDs.

Commonly, after the surface permanent magnet motor is analyzed by the 3-D finite element method, we can observe the magnetic field distribution map on the motor's surface, as shown in Fig. 7. Even if the stereoscopic 3-D monitor [6] is used to visualize the magnetic field distribution map like Fig. 7, the magnetic field inside the motor can't be observed. Consequently, the visualization method using the volumetric display is superior to the other visualizations. However, the developed volumetric display has just two LCDs, so it is necessary to increase the number of LCDs in order to observe in detail.

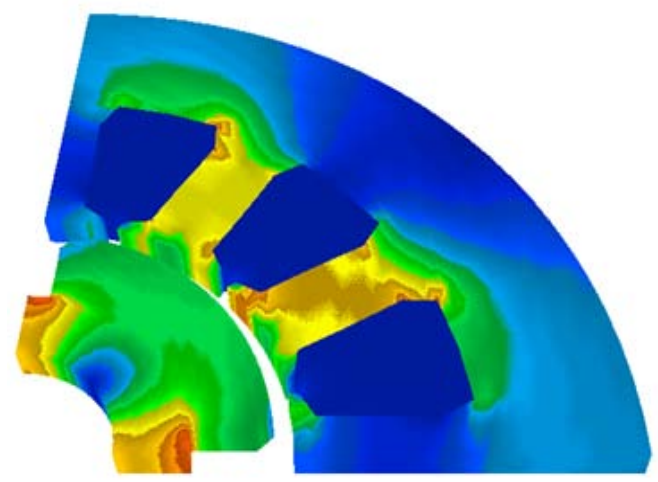

Fig. 7. The common magnetic field distribution map on the surface of the surface permanent magnet motor. Even if the stereoscopic 3-D monitor is employed, the magnetic field inside the object can't be observed.

\section{COMPARISON OF IMAGES}

The simulation results are often compared, for design, improving performance, and so on. Usually, in order to compare images, these images are overlappingly displayed on a monitor, as shown in Fig. 8. However, some blue cones are hidden by the red ones, so the detail of the difference can't be observed.

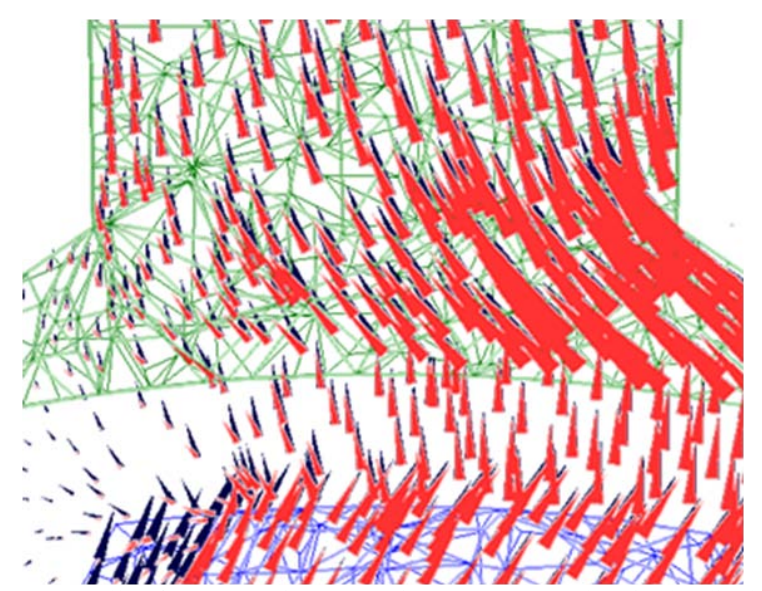

Fig. 8. Usually, for comparison, two images are overlappingly displayed. However, some blue cones are hidden by the red ones.

The volumetric display is effective in the image comparison. When the LCDs of the volumetric display are close each other, all the images on every LCD are clearly perceived. Consequently, it is easy to observe even a small difference between the images on the front and rear LCDs. The image on the rear LCD may be less-visible due to overlapping images, however we can clearly observe the rear image by moving our eye's point, as shown in Fig. 9. 


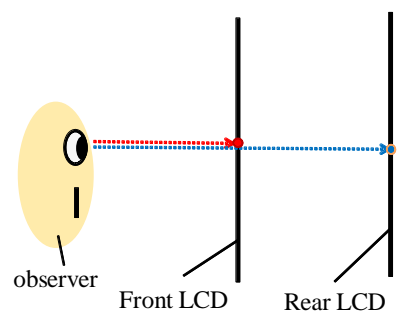

(a)

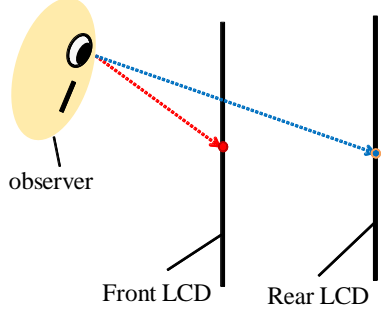

(b)
Fig. 9. With the volumetric display, two images are overlappingly observed. It is possible to observe the object, which is hidden in (a), by moving the eye's point, as shown in (b).

An example of the image comparison with volumetric display is shown in Fig. 10. Observing the images on LCDs from the right front, as shown in Fig. 10(a), the overlapping image can be perceived, like Fig. 8. Some hidden blue cones are invisible, however we can perceive the overlapping part by becoming black. In addition, when moving the eye's point, the hidden blue cones appear clearly, so we can easily compare the two images with less labor.

\section{CONCLUSION}

The visualization technology is undeveloped though the 3-D analysis technology is rapidly developed. If the result of the 3$\mathrm{D}$ analysis couldn't be observed in detail, the 3-D analysis might be useless or ineffective. For a high degree of design, the 3-D observation is the most effective method.

We have developed the volumetric display. It consists of two LCDs. Depicting the magnetic field vector maps on each $\mathrm{LCD}$, it is possible to observe the semi-three-dimensional view. We can recognize the distance to the image on each LCD, so that the magnetic field inside the object can be perceived three-dimensionally. It was confirmed that the volumetric display was effective in the observation of the magnetic field in 3-D space.

In addition, the comparison of the magnetic field was performed using the volumetric display. It is possible to clearly observe the back cones, when moving the eye's point. It was also confirmed that the volumetric display was effective in the comparison of the magnetic fields.

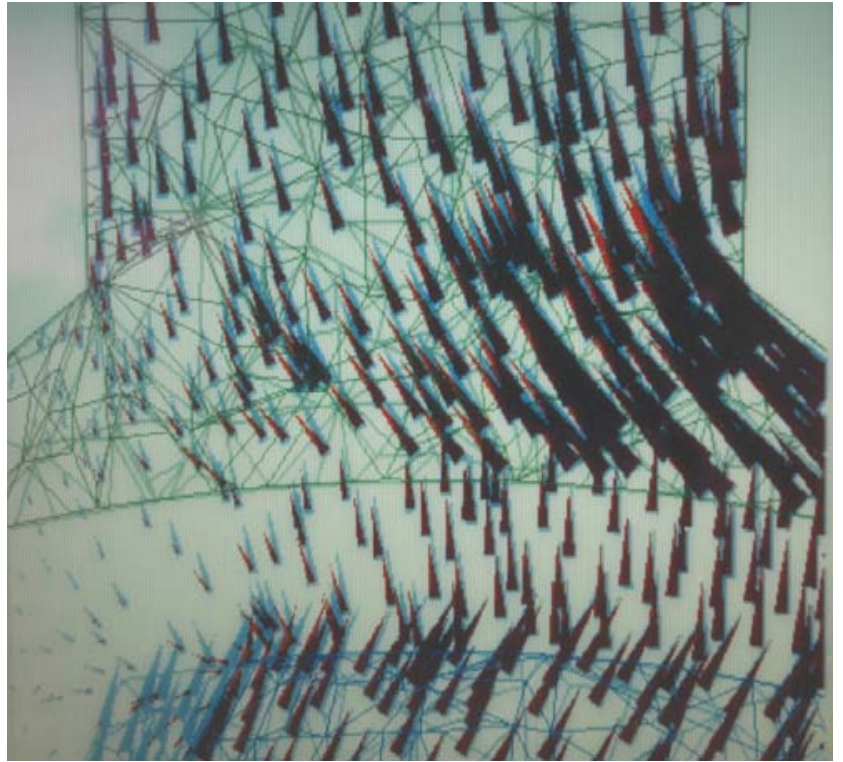

(a)

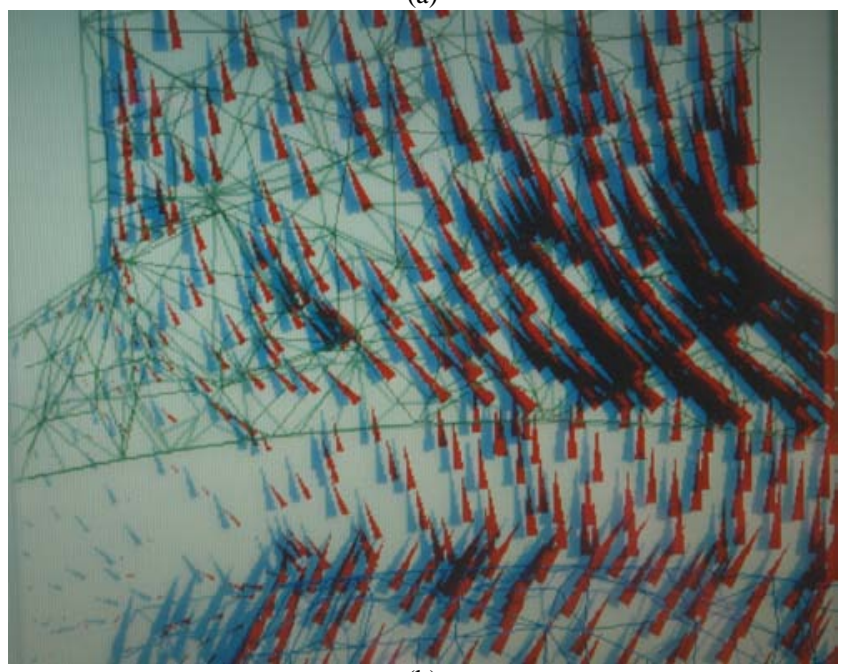

(b)

Fig. 10. The image comparison with the volumetric display. (a) The picture of the LCDs viewed from the right front. (b) The picture of the LCDs viewed from the different angle. When changing the view angle, the hidden blue cones appear.

\section{REFERENCES}

[1] E. P. Furlani, Permanent Magnet and Electromechanical Devices: Materials, Analysis and Applications, Academic Press, New York, 2001.

[2] J. P. A. Pedro and N. Sadowski, ELECTROMAGNETIC MODELING BY FINITE ELEMENT METHODS, Marcel Dekker, New York, 2003.

[3] S. Noguchi, T. Yoshigai, and H. Yamashita, “Analytical Computation and Visualization of Magnetic Flux Lines in 3-D Space From Hexahedral Edge Finite Element Results," IEEE Transactions on Magnetics, vol. 41, no. 5, pp. 1820-1823, May 2005.

[4] M. Hafner, M. Schoning, M. Antczak, A. Demenko, and K. Hameyer, "Methods for efficient computation and visualization of magnetic flux lines in 3D,” Proceedings of COMPUAG 2009, pp. 622-623, Nov. 2009.

[5] S. Noguchi and H. Yamashita, "Visualization Method of Magnetic Flux Lines with Accurate Allocation Applying Tube System,” Proceedings of COMPUAG 2009, pp. 867-868, Nov. 2009.

[6] http://www.sharp.co.jp/lcd-display/crisia/lineup/ll-151d/index.html

[7] A. Sullivan, "A Solid-state Multi-planar Volumetric Display," The Society for Information Display, Digests of Technical Paper, SID 2003, p. 1531, 2003.

[8] http://www.lightspacetech.com/

[9] http://actuality-medical.com/site/content/perspecta_display1-9.html 